Results ESC sudden cardiac death risk scores were comparable between the HCM groups (HCM:2.2 $\pm 1.5 \%$, HCM-DM:1.9 $\pm 1.2 \% ; \mathrm{p}=\mathrm{NS})$ and sarcomeric mutations were equally common. HCM-DM had the highest NT-proBNP levels (HV:42 ng/L[IQR:35-66 ng/L], DM:118 ng/L[IQR:53-187 ng/L], HCM:298 ng/L[IQR:157-837 ng/L], HCM-DM:726 ng/L [IQR:213-8695 ng/L]; $\mathrm{p}<0.0001)$. Left-ventricular ejection fraction, mass and wall thickness were similar between the HCM groups. HCM-DM displayed a greater degree of fibrosis burden with higher extracellular volume fraction and scar percentage, more significant reductions in global longitudinal strain and left atrial function compared to the isolated HCM. PCr/ATP was similarly decreased in the HCM-DM and DM (HV:2.17 \pm 0.49 , DM:1.58 \pm 0.27, HCM:1.93 \pm 0.38 , HCMDM:1.54 $\pm 0.27 ; \mathrm{p}=0.0002)$. HCM-DM had the lowest stress myocardial blood flow (HV:2.06 $\pm 0.42 \mathrm{ml} / \mathrm{min} / \mathrm{g}$, DM:1.95 $\pm 0.41 \mathrm{ml} / \mathrm{min} / \mathrm{g}$, HCM:1.74 $\pm 0.44 \mathrm{ml} / \mathrm{min} / \mathrm{g}$, HCM-DM:1.39 $\pm 0.42 \mathrm{ml} / \mathrm{min} / \mathrm{g} ; \mathrm{p}=0.0017$ ).

Conclusions Comorbid diabetes adversely affects the HCM phenotype with greater reductions in myocardial energetics, perfusion, strain, increased scar burden and higher NT-proBNP levels compared to isolated HCM. Our findings suggest that specific, targeted therapeutic approaches may be useful in hypertrophic cardiomyopathy patients with diabetes comorbidity to improve clinical outcomes.

\section{THE ROLE OF CARDIAC MRI IN ASSESSING BICUSPID AORTIC VALVE DISEASE AND ASSOCIATED MYOCARDIAL FIBROSIS}

Nikhil Chatrath, Raghav Bhatia, Saad Fyyaz, Hamish Maclachlan, Sanjay Sharma, Maite Tome, Michael Papadakis. St George's University, London

\subsection{6/heartjnl-2021-BSCMR.15}

Introduction Bicuspid aortic valve (BAV) is the most common congenital cardiovascular abnormality. Cardiac MRI (CMR) is commonly used to screen for aortic dilatation and associated abnormalities. Sudden death has been reported, often due to aortic stenosis (AS) or dissection, but it has been hypothesised that areas of fibrosis may be a substrate for arrythmias in patients with BAV.

Methods The aim of this study was to review the CMR findings of consecutive patients with BAV including patterns of late gadolinium enhancement (LGE). Valve morphology was described according to recent international guidance ${ }^{1}$. The prevalence of aortic dilatation, left ventricular hypertrophy $(\mathrm{LVH})$, AS, aortic regurgitation (AR) and the distribution of late gadolinium enhancement (LGE) was noted.

Results 217 patients with BAV had CMR performed at a single-centre between May 2011 and September 2021; mean age 46 years (IQR 32-62), 74.6\% male $(n=162)$. 6.5\% $(n=14)$ had an aortic valve replacement (AVR). 25.8\% $(n=56)$ had at least moderate AS and $17.5 \%(n=38)$ at least moderate AR, quantified by flow measurements.

Valve morphology could be established in 161 cases; $89.4 \%$ $(n=144)$ demonstrated a fused BAV, the majority demonstrating right-left cusp fusion $(73 \% ; n=105) .164$ had a full aortogram performed, of which $9.8 \% \quad(n=16)$ had evidence of aortic coarctation. When indexed to body surface area, $28.7 \%$ $(n=47)$ had dilatation at the sinus of Valsalva, $39.0 \%$ $(n=64 \%)$ at the sino-tubular junction and 56.2\% $(n=94)$ at the ascending aorta.
Delayed enhancement images were acquired in 116 studies and LGE was identified in $20.7 \%(n=24)$ of these. In $45.8 \%$ $(n=11)$, the LGE could be attributed to other causes, most commonly infarction, with significant coronary artery disease confirmed on angiography $(n=9)$. In the remaining subjects, none of whom had evidence of AS or LVH, there was midwall LGE in 13 subjects; predominantly in the basal septum $(n=7)$, lateral wall $(n=3)$, inferior RV insertion point $(n=2)$ and anterior wall $(n=1)$.

Conclusion This study highlights the multiple roles of CMR in the assessment of individuals with BAV. Further larger-scale studies are required to analyse patterns of LGE to try and better understand the distribution of fibrosis in these patients, and to correlate these findings with clinical outcomes.

\section{REFERENCE}

1. Michelena HI, Della Corte A, Evangelista A, et al. International consensus statement on nomenclature and classification of the congenital bicuspid aortic valve and its aortopathy, for clinical, surgical, interventional and research purposes. J Thorac Cardiovasc Surg. 2021;162(3):e383-e414. doi:10.1016/j. jtcvs.2021.06.019

\section{VALIDATION OF TIME-RESOLVED, AUTOMATED PEAK TRANSVALVULAR VELOCITY TRACKING THROUGH THE MITRAL VALVE USING FOUR-DIMENSIONAL FLOW CARDIOVASCULAR MAGNETIC RESONANCE}

\begin{abstract}
${ }^{1,2}$ Paul Njoku, ${ }^{2}$ Ciaran Grafton-Clarke, ${ }^{1}$ Hosam Assadi, ${ }^{3}$ Rebecca Gosling, ${ }^{3}$ Gareth Archer ${ }^{1,2}$ Marcus Flather, ${ }^{1,2}$ Vassilios Vassiliou, ${ }^{1,2}$ Pankaj Garg. ${ }^{1}$ University of East Anglia, Norwich Medical School, Norfolk, UK; ${ }^{2}$ Norfolk and Norwich University Hospitals NHS Foundation Trust, Norfolk, UK; ${ }^{3}$ University of Sheffield Medical School and Sheffield Teaching Hospitals NHS Trust, Sheffield, UK
\end{abstract}

\subsection{6/heartjnl-2021-BSCMR.16}

Doppler echocardiography (TTE) remains the imaging modality of choice for the assessment of mitral inflow and left ventricular diastolic function, despite its limitations. Four-dimensional flow cardiovascular magnetic resonance (4D flow CMR) offers time-resolved cross-sectional velocity data, which can be used to investigate transvalvular peak velocity through the mitral valve. This would not suffer from the in-plane motion and angle-dependence of pulsewave echocardiography.

Objective We aim to validate a novel time-resolved, automated dynamic 4D flow CMR peak velocity tracking method for measuring the peak velocity of mitral inflow against TTE.

Method Patients recruited to EurValve programme $(n=22)$ underwent TTE and 4D flow CMR. Peak E-wave and A-wave velocities were recorded. This work was done in collaboration with the industry leader in 4D flow CMR (PIE Medical Imaging). Transvalvular flow segmentation was done using established valve tracking methods and the generated $3 \mathrm{D}$ streamlines were investigated for seeking the peak velocity inside the left ventricular cavity during diastole. Reproducibility analyses were carried out in 10 cases.

Results The peak E-wave mitral inflow velocity was comparable between the novel 4D flow method and TTE $(1.09 \pm$ $0.29 \mathrm{~m} / \mathrm{s}$ and $1.10 \pm 0.37 \mathrm{~m} / \mathrm{s}$ respectively; $\mathrm{p}=0.60)$. The mean A-wave peak velocity was also comparable across both methods $(0.94 \pm 0.40 \mathrm{~m} / \mathrm{s}$ and $0.86 \pm 0.29 \mathrm{~m} / \mathrm{s}$ respectively; $\mathrm{p}=0.38$ ). The automated $4 \mathrm{D}$ flow method also showed good correlation with TTE for both E-wave $(\mathrm{r}=0.54 ; \mathrm{p}=0.01)$ and A-wave $(\mathrm{r}=0.55 ; \mathrm{p}=0.03)$ with 

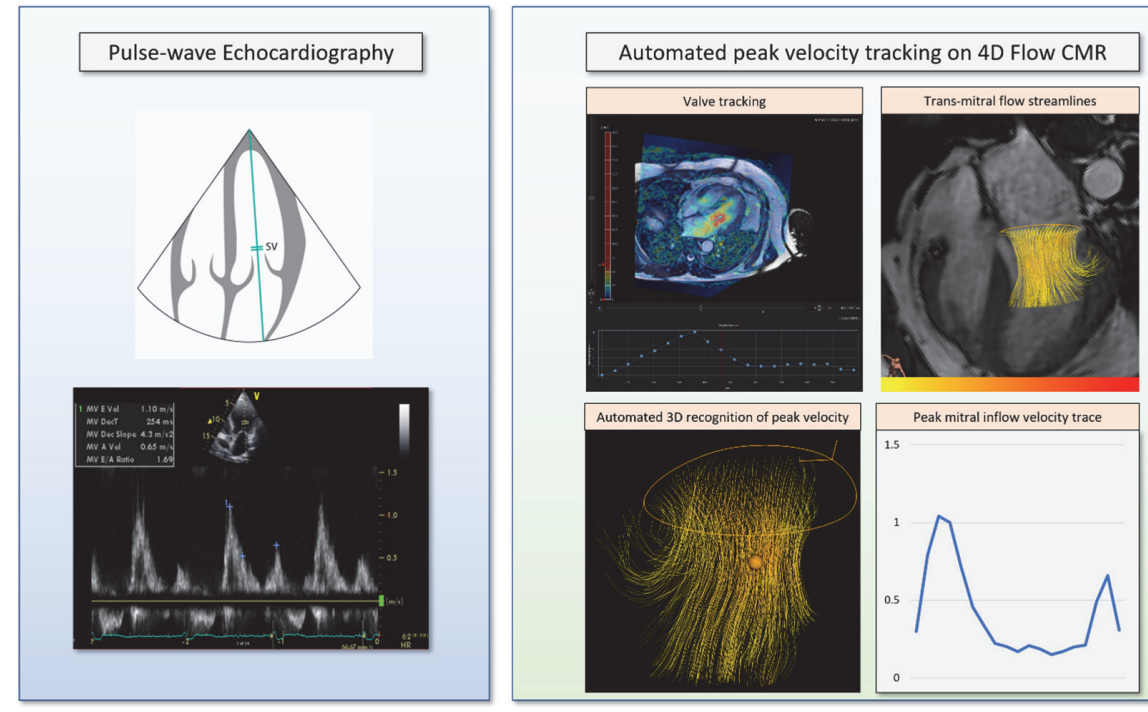

Automated 30 recognition of peak velocity
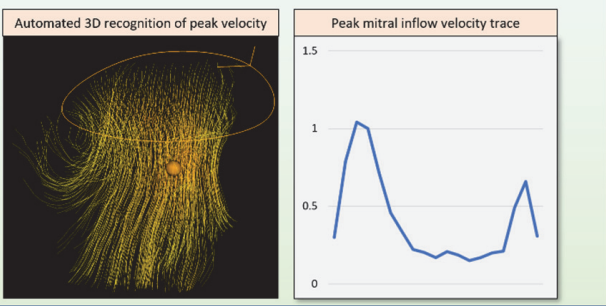

Abstract 16 Figure 1 Automated peak velocity tracking using 4D flow CMR was validated in 22 patients against pulse-wave Doppler Echocardiography for mitral inflow assessment
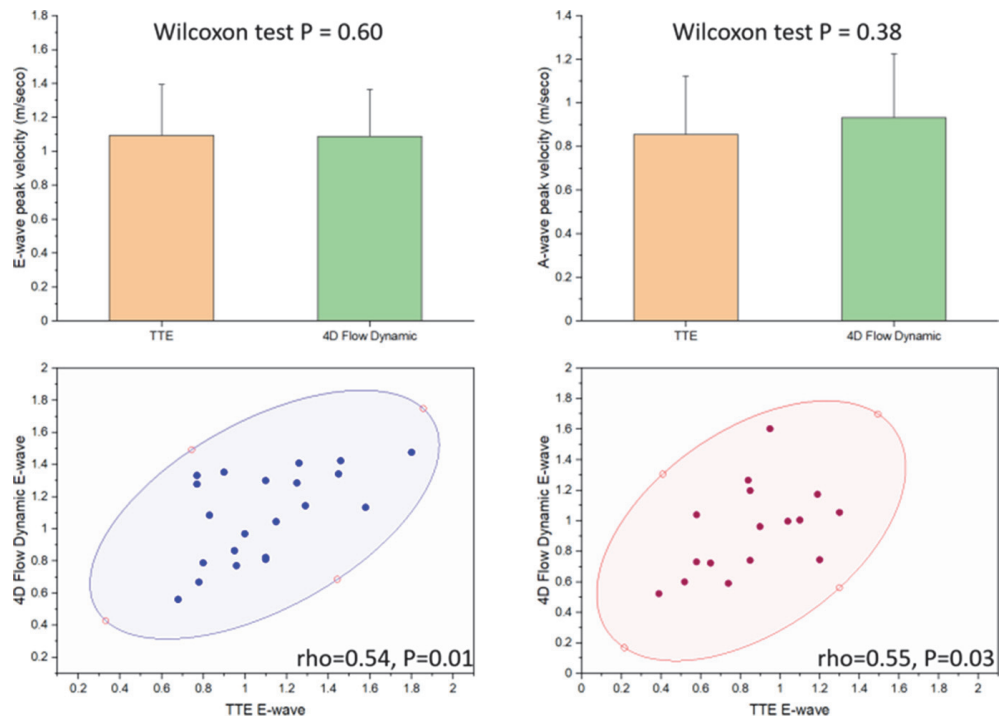

Abstract 16 Figure 2 Mean bar chart and scatter plots demonstrating agreement between PW-Doppler and novel automated 4D flow CMR method
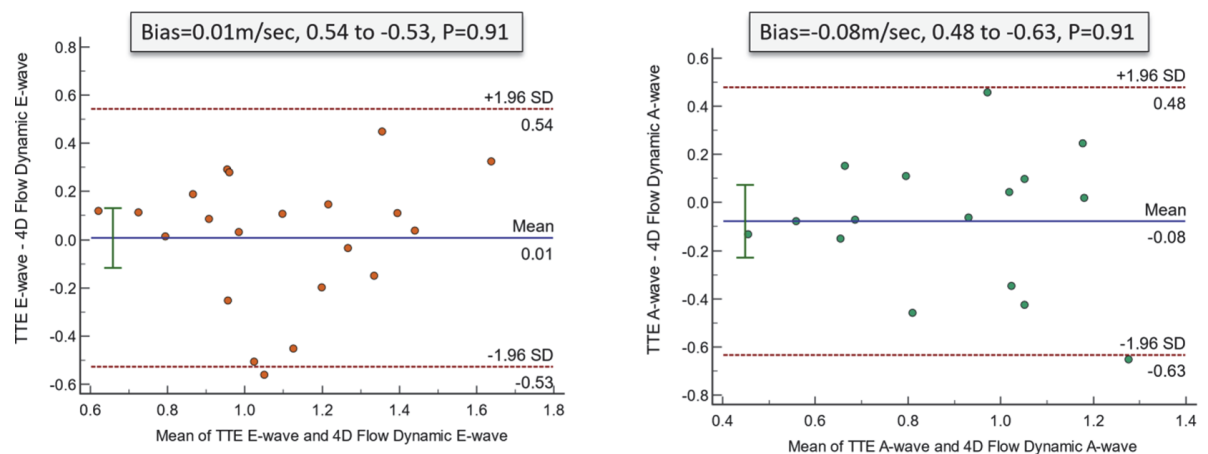

Abstract 16 Figure 3 Bland-Altman plots demonstrate minimal and statistically non-significant bias between PW-Doppler and novel 4D flow method for peak mitral inflow assessment 
minimal and non-significant bias between the two modalities (bias $=0.01 \mathrm{~m} / \mathrm{s} ; \mathrm{p}=0.91$ and $-0.08 \mathrm{~m} / \mathrm{s} ; \mathrm{p}=0.91$ ). This novel automated method demonstrated excellent reproducibility (Coefficient of variability $2.67 \%$ for peak E-wave mitral inflow velocity; Coefficient of variability $1.93 \%$ for peak A-wave mitral inflow velocity).

Conclusion We present a novel automated time-resolved transvalvular peak velocity assessment solution that can be used clinically for mitral inflow assessment and would circumvent the limitations of pulse-wave doppler echocardiography. Future studies are warranted to explore the diagnostic and prognostic advantages of our novel automated technique for mitral inflow assessment.

\section{PARTICIPANTS WITH DIABETES MELLITUS HAVE PRESERVED METABOLIC FLEXIBILITY}

Peregrine Green, William D Watson, Neil Herring, Stefan Neubauer, Oliver J Rider. Oxford Centre for Clinical Magnetic Resonance Research, Division of Cardiovascular Medicine, Radcliffe Department of Medicine, University of Oxford, UK; Department of Physiology, Anatomy and Genetics, University of Oxford, UK

\subsection{6/heartjnl-2021-BSCMR.17}

Background Measurement of the Phosphocreatine/Adenosine Triphosphate (ATP) ratio along with the Creatine Kinase (CK) rate constant $\left(\mathrm{CK}_{k f}\right)$ allows calculation of the ATP delivery rate (CK flux). Metabolic flexibility may be impaired both in heart failure with reduced ejection fraction (HFrEF) and diabetes mellitus (DM). It is unknown to what extent flexibility can be influenced by artificially altering the substrate available for metabolism.

Purpose To examine cardiac function and energetics in diabetic participants with normal cardiac function and HFrEF, clamped on either fatty acid (FA) or glucose metabolism.

Methods Participants with non-insulin dependent diabetic mellitus (NIDDM) with both normal cardiac function (NHDM) and HFrEF (HFDM) were recruited and received intravenous infusions of either Intralipid (IL) or glucose-insulin (GI) at 2 separate visits, before undergoing multi-parametric cardiac MRI at 3 Tesla. Cardiac volume and function, PCR/ATP and $\mathrm{CK}_{k f}$ were assessed. CK flux was calculated as $\mathrm{CK}_{k f} \times \mathrm{PCR} /$ ATP $\times 5.7 \quad \mu \mathrm{mol} \quad(\mathrm{g} \text { wet weight })^{-1}$ (assumed ATP concentration).

Results 15 NHDM participants (14 male, age $61.5 \pm 7.3$ years) and 9 HFDM participants (7 male, age $69.4 \pm 7.8$ years) were recruited. Left ventricular ejection fraction (LVEF) at rest was higher on IL compared to both baseline fasting and GI for NHDM (baseline 59.1 $\pm 3.8 \%$, GI 59.4 $\pm 4.3 \%$, IL $62.8 \pm 3.5 \% ; \mathrm{p}=<0.01$ ), with a non-significant trend for HFDM (baseline $37.3 \pm 7.6 \%$, GI $36.8 \pm 9.2 \%$, IL $38.8 \pm 8.0 \%$, $\mathrm{p}=0.12)$. For both NHDM and HFDM there was no difference in PCR/ATP (NHDM: GI 1.98 \pm 0.31 , IL 1.97 \pm 0.24 , $\mathrm{p}=0.99$; HFDM: GI 1.82 \pm 0.36 , IL 2.01 $\pm 0.32, \mathrm{p}=0.09$ ) or CK flux (NHDM: GI 2.6 $\pm 1.1 \mu \mathrm{mol}$ (g wet weight) ${ }^{-1} \mathrm{~s}^{-1}$, IL $1.8 \pm 1.2 \mu \mathrm{mol}$ (g wet weight) ${ }^{-1} \mathrm{~s}^{-1}, \mathrm{p}=0.08$; HFDM: GI 1.6 $\pm 1.7 \mu \mathrm{mol}(\mathrm{g} \text { wet weight) })^{-1} \mathrm{~s}^{-1}$, IL $2.3 \pm 1.1 \mu \mathrm{mol}$ (g wet weight) ${ }^{-1} \mathrm{~s}^{-1}, \mathrm{p}=0.39$ ).

Conclusion Diabetic participants with HFrEF and normal cardiac function appear to have increased resting LVEF when clamped on FA as opposed to glucose metabolism, without a significant change in energetic status. This may imply that metabolic flexibility is relatively preserved in these groups.

\section{SUBCLINICAL MYOCARDIAL INFLAMMATION IN ADULTS WITH TYPE 2 DIABETES: A CLINICAL STUDY USING MYOCARDIAL T2 MAPPING}

Pranav Ramesh, Jian L Yeo, Gaurav S Gulsin, Gerry P McCann. Department of Cardiovascular Sciences, University of Leicester and Leicester NIHR Biomedical Research Centre, Glenfield Hospital, Leicester, UK

\subsection{6/heartjnl-2021-BSCMR.18}

Background Chronic hyperglycaemia in Type 2 diabetes (T2D) results in a systemic low-grade inflammatory state. Inflammation is a key instigator in the development of heart failure in T2D. Cardiovascular magnetic resonance (CMR) T2 mapping is a technique which identifies myocardial oedema. The utility of T2 mapping to identify subclinical oedema as a marker of inflammation in T2D is unknown. We hypothesise that T2 times will be higher in subjects with T2D.

Methods CMR imaging on a 3-Tesla scanner was performed on 182 participants who were free of symptomatic cardiovascular disease. T2 images were acquired using the Siemens MyoMap sequence at the mid-ventricular short-axis slice. Twenty participants underwent a repeat CMR scan within two weeks to assess the test-retest reproducibility of T2. Intraclass correlation coefficient (ICC) and Bland-Altman plots were generated to assess reproducibility. T2 values between groups were compared using T-test or Mann-Whitney test as appropriate. Clinical determinants of $\mathrm{T} 2$ in $\mathrm{T} 2 \mathrm{D}$ were assessed using multivariable linear regression.

Results 124 T2D (mean age 64 7 , 66\% male) and 40 controls (mean age $61 \pm 8,60 \%$ male) were analysed. T2 times exhibited excellent intra-observer (ICC 0.98-0.99), moderate inter-observer (ICC 0.48-0.99), and poor test-retest variability (ICC 0.33-0.90). T2 times were significantly lower in subjects with T2D compared to controls $(39.0 \pm 2.2 \mathrm{~ms}$ versus 40.1 $\pm 2.9 \mathrm{~ms}, \mathrm{P}=0.013)$. Stratification by sex revealed significantly lower T2 in females with T2D $(39.4 \pm 2.4$ ms versus $41.7 \pm 3.1$ $\mathrm{ms}, \mathrm{P}=0.003)$, but not in males, when compared to controls. Following multivariable adjustment, T2 time was positively associated with a non-white ethnicity $(\beta=0.245, \mathrm{P}=0.007)$ and diabetic duration $(\beta=0.197, P=0.03)$ and inversely associated with systolic blood pressure $(\beta=-0.215, \mathrm{P}=0.018)$.

Conclusions T2 mapping has moderate-excellent observer variability but poor test-retest reproducibility in a cohort T2D. Lower T2 times in T2D may reflect early myocardial fibrosis but does not provide evidence of subclinical myocardial oedema and therefore is not able to detect low-grade myocardial inflammation.

\section{CARDIAC MAGNETIC RESONANCE TO IDENTIFY RAISED LEFT VENTRICULAR FILLING PRESSURE}

Rebecca Gosling, Samer Alabed, Peter Swoboda, Sherif F Nagueh, Rachel Jones, Alexander Rothman, Jim M Wild, David G Kiely, Robin Condliffe, Andrew J Swift, Pankaj Garg.

\subsection{6/heartjnl-2021-BSCMR.19}

Background Non-invasive imaging is routinely used to estimate left ventricular (LV) filling pressures (LVFP) in heart failure (HF), as an alternative to right heart catheterisation (RHC). Transthoracic echocardiography (TTE) estimates of LVFP are frequently deployed but produce largely dichotomised data limiting flexible clinical use and perform less well in patients with heart failure with preserved ejection fraction (HFpEF). 